\title{
Effects of Implementing an Ergonomic Work Schedule on Heart Rate Variability in Shift-working Nurses
}

\author{
Susanna Järvelin-Pasanen ${ }^{1}$, Annina Ropponen ${ }^{2}$, Mika TARVAinen ${ }^{3,4}$, \\ Marja Paukkonen ${ }^{5}$, Tarja Hakola ${ }^{6}$, Sampsa Puttonen ${ }^{6,7}$, Pasi Antero Karjalainen ${ }^{3}$, \\ Harri LindHolm ${ }^{6}$, Veikko LouhevaARA ${ }^{1}$ and Tiina PoHJonen ${ }^{8}$
}

${ }^{1}$ Ergonomics, Institute of Public Health and Clinical Nutrition, School of Medicine, Faculty of Health Sciences, University of Eastern Finland, Finland, ${ }^{2}$ Institute of Biomedicine, School of Medicine, Faculty of Health Sciences, University of Eastern Finland, Finland, ${ }^{3}$ Department of Applied Physics, Faculty of Science and Forestry, University of Eastern Finland, Finland, ${ }^{4}$ Department of Clinical Physiology and Nuclear Medicine, Kuopio University Hospital, Finland, ${ }^{5}$ Health Centre, City of Helsinki, Finland, ${ }^{6}$ Finnish Institute of Occupational Health, Finland, ${ }^{7}$ Institute of Behavioural Sciences, University of Helsinki, Finland and ${ }^{8} \mathrm{Helsinki}$ City Occupational Health Centre, Finland

\begin{abstract}
Effects of Implementing an Ergonomic Work Schedule on Heart Rate Variability in Shiftworking Nurses: Susanna Järvelin-PASANEN, et al. Ergonomics, Institute of Public Health and Clinical Nutrition, School of Medicine, Faculty of Health Sciences, University of Eastern Finland-Objectives: The aim of this study was to compare the psychophysiological strain related to a conventional shift schedule and new ergonomically improved two- and three-shift schedules using heart rate variability (HRV) analysis. The specific aim was to determine whether the introduced ergonomic shift arrangement had any positive effects on the psychophysiological strain such as increased HRV or decrease in the sympathovagal balance of the autonomic nervous system (ANS). Methods: Questionnaire data and 24-hour HRV recordings were gathered from 48 female shift-working nurses once while working the conventional shift schedule (baseline) and again after one year working an ergonomic shift schedule during the morning shift. Results: Comparisons between conventional and ergonomic shift schedules (baseline and follow-up, respectively) revealed significant differences in frequency-domain parameters. Implementing an ergonomic shift schedule resulted in decreased normalized low frequency (LF) power, increased normalized high frequency (HF) power, and decreased LF/HF ratio at the beginning of the shift. Furthermore, at baseline, mean RR interval, root mean square of successive RR interval differences (RMSSD)
\end{abstract}

Received Oct 30, 2012; Accepted Apr 11, 2013

Published online in J-STAGE May 13, 2013

Correspondence to: S. Järvelin-Pasanen, Ergonomics, Institute of Public Health and Clinical Nutrition, School of Medicine, Faculty of Health Sciences, University of Eastern Finland, P.O. Box 1627, FI-70211 Kuopio, Finland and normalized HF power were increased at the end of the shift compared with the values at the beginning of the morning shift. In contrast, at the follow-up, LF power was increased between the end and beginning of the morning shift. Conclusions: The psychophysiological strain measured by HRV analysis was lower at the beginning of the work shift for the ergonomic shift schedules compared with the conventional schedule. This indicates that an ergonomic shift schedule may have a positive effect on the ANS recovery occurring between successive work shifts.

(J Occup Health 2013; 55: 225-233)

Key words: Autonomic nervous system, Heart rate variability, Shift work, Scheduling

Shift work seems to exert a wide range of detrimental health effects ${ }^{1-4)}$. It has been shown to increase the risk of cardiovascular diseases ${ }^{1,3,5)}$, gastrointestinal disorders and diseases, breast cancer, and type II diabetes ${ }^{4)}$ and metabolic syndrome ${ }^{6)}$. In addition, shift work may be related to disturbances in psychological well-being and mental health ${ }^{4}$, and may confer a higher risk of sleep disorders, fatigue ${ }^{1,2,4)}$ and occupational accidents ${ }^{1,7)}$. However, if one wishes to decrease the negative consequences of shift work, then one needs knowledge of working time arrangements that would promote health.

It has been claimed that changes in psychophysiological strain (i.e., the strain related to shift work) and recovery of the autonomic nervous system (ANS) can be evaluated by heart rate variability (HRV) analyses ${ }^{8,9)}$. Shift work disturbs the normal circadian rhythm of HRV. Previous studies have shown that shift work acutely increases heart rate and 
blood pressure, and the normal circadian rhythm of HRV is powerfully modified by the physical activity exerted during the night shift ${ }^{10,11)}$. In addition, shift work has been shown to be associated with reduced $\mathrm{HRV}^{12-14)}$. Decreases in specific HRV parameters that reflect parasympathetic activity of the ANS have been observed among shift-working female nurses ${ }^{12)}$ and other shift workers ${ }^{13,14)}$. These unfavorable changes in HRV may partially explain the increased cardiovascular risk and cardiovascular morbidity in shift workers ${ }^{15,16)}$

Previous research of shift work with regard to HRV has focused on comparison with daytime work $^{10,12-14,17-19)}$, and changes in psychophysiological function after modifying shift schedules warrant further studies.

Health and social problems caused by shift work could be prevented or minimized by the implementation of ergonomic recommendations ${ }^{20,21)}$ (Table 1). For example, a controlled intervention study with male technicians showed that a rapidly forward-rotating shift system had positive effects on sleep, alertness and well-being, especially in older shift workers ${ }^{22}$, and similar effects have been seen in steel workers ${ }^{23)}$. However, as far as we are aware, the effects of ergonomic working time arrangements have not been stud- ied with regard to objective measures of strain and recovery in shift work ${ }^{24)}$.

The aim of this study was to compare psychophysiological strain related to conventional and ergonomic shift schedules in a within-subject one year beforeafter design. The specific aim was to investigate if a switch to a more ergonomic shift arrangement would have any positive effects on the psychophysiological strain as reflected by either increased parasympathetic activity or decreased sympathovagal balance of the ANS.

\section{Subjects and Methods}

The subjects were female and male nurses $(n=90)$ who voluntarily participated in the Healthy Working Hours Research and Development Project ${ }^{25)}$. They worked on a three-shift (including a morning shift, from 07:00 a.m. to 02:30 p.m.; evening shift, from 01:00 to 09:00 p.m.; and night shift, from 09:00 p.m. to 07:30 a.m.) or two-shift system (including morning and evening shifts but no night shift) in six different wards providing either acute or long-term care in municipal hospitals. Subjects with missing $(n=29)$ or poor $(n=7)$ HRV data were excluded from the analyses. Furthermore, the male subjects $(n=6)$ were excluded. Hence, the final sample comprised of 48

Table 1. The ergonomic guidelines for shift work ${ }^{20,21)}$

\begin{tabular}{l} 
Recommendations \\
\hline 1. Few night shifts in succession \\
2. Avoid permanent night work \\
3. Few morning shifts in succession \\
4. Few evening shifts in succession \\
5. Forward rotation \\
6. At least 2 days off after last night shift \\
7. Avoid single days off between night shifts \\
8. Avoid single working days between days off \\
9. Maximum of $5-7$ working days \\
10. Extended shifts ( $>8$ hours) are only acceptable if the nature of work and workload are suitable, and complete \\
recovery after work is possible \\
11. There should be adequate resting time ( $>11$ hours) between two shifts \\
12. Start of morning shift not too early \\
13. End of evening shift not too late \\
14. End of night shift as early as possible \\
15. Avoid work on weekends \\
16. Some free weekends with at least 2 consecutive days off \\
17. Avoid short-term deviations from set shift schedule \\
18. "Rules of the game" concerning time of advance notice and compensation \\
19. Co-workers fix their working time themselves and make themselves responsible for doing their tasks on time \\
20. Make flexibility possible \\
\end{tabular}


female nurses with HRV data from the morning shift (Fig. 1). Each subject filled in a written informed consent form. The study protocols were approved by the Ethics Committee of Helsinki University Hospital and the Research Committee of Health Centre, Helsinki (HUS 265/E0/05). The study was conducted according to the ethical standards of the Declaration of Helsinki.

\section{Study design}

The data were gathered through 24-hour HRV recordings supplemented with a questionnaire both before the implementation of changes to shift-work schedules (baseline period of September-November 2005, later referred to as the conventional shift schedule) and about one year after the changes (follow-up on December 2006, ergonomic shift schedules). In this prospective study, each subject served as her own control.

As a part of the Healthy Working Hours Research and Development Project ${ }^{25}$, the shift-work schedules were altered to meet the recommended ergonomic guidelines $^{20,21)}$. Because of the irregular and slightly individual shift-work schedules of nurses, the main alteration was from backward to forward rotation. This was implemented by reducing quick returns (i.e., less morning shifts immediately after evening shifts). The number of total working hours (114 hours 45 minutes) and the amounts of weekend work (one out of three free), evening work, and night work (depending on ward) per 3-week period remained unchanged $^{25)}$.

\section{Heart rate variability recordings}

The 24-hour HRV recordings were performed twice, at baseline while the nurses were working on the conventional shift schedule and in the follow-up after the implementation of the more ergonomic schedules. The time between the baseline and follow-up measurements was about one year.

All HRV recordings were performed during the morning shifts (from 7:00 a.m. to 2:30 p.m.). The recording periods started before the beginning of the work shift and ended about 24 hours later. Thus, the HRV recordings included the actual work shift, leisure time and the night after the work shift. Throughout the 24-hour recording period, the subjects were instructed to work in their habitual manner and to maintain their normal lifestyle. There were no diaries or other measures for the recording periods available for this study.

The HRV recordings were performed between successive work shifts. Thus, when analyzing HRV, the previous work shift has to be taken into account, which were the morning shift $(n=18$ and 22$)$, evening

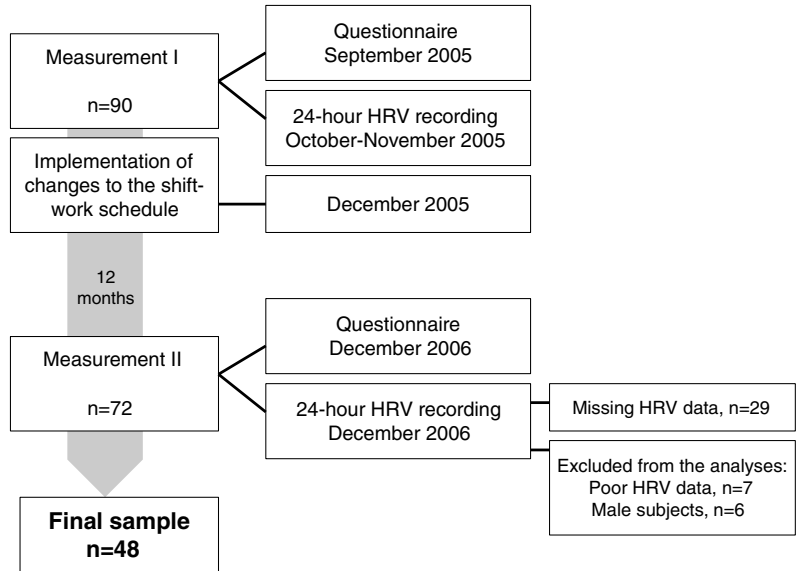

Fig. 1. The study design and number of subjects. HRV: heart rate variability.

shift ( $n=18$ and 4) or day off ( $n=6$ and 15). The information about the previous shift was not available in six and seven cases (at baseline and follow-up, respectively).

The beat-to-beat heart rate (HR) was measured with a Suunto T6 (at baseline) or Suunto Smart Belt (at follow-up) HR monitor (Suunto Ltd., http://www. suunto.fi). The recording of HRV with an HR monitor has been shown to be valid and reliable during physically and mentally stressful conditions, especially when chest electrodes are used ${ }^{26}$.

Three time-domain and five frequency-domain HRV parameters were selected for the study as a way of obtaining an overall view of HRV with a minimum set of standardized parameters ${ }^{8}$. The timedomain parameters were the mean of beat-to-beat RR interval series (meanRR), the standard deviation of all normal RR intervals (SDNN), and the root mean square of successive RR interval differences (RMSSD). MeanRR is inversely proportional to mean heart rate. SDNN demonstrates overall HRV (both sympathetic and parasympathetic control) and its cyclic components, whereas RMSSD reflects mainly parasympathetic control of heart rate. The frequencydomain parameters were the absolute powers of the low-frequency (LF: $0.04-0.15 \mathrm{~Hz}$ ) and high-frequency (HF: $0.15-0.4 \mathrm{~Hz}$ ) components, normalized powers of LF and HF components and the ratio between LF and $\mathrm{HF}$ powers (LF/HF ratio). The LF power consists of both sympathetic and parasympathetic control, and HF power is associated with respiration-related heart rate changes and demonstrates parasympathetic control of heart rate. LF and HF powers in normalized units represent the relative power in proportion to the total power minus the power of the very low frequency (VLF: $0-0.04 \mathrm{~Hz}$ ) component. The $\mathrm{LF} / \mathrm{HF}$ ratio is commonly used as an index of sympathovagal balance. 
In the first part of the HRV analyses, the raw HR data of both measurements were carefully visually inspected by the researcher in order to exclude artefacts. Two artefact-free $10-$ min time periods from both 24-hour recordings were systematically selected for the analyses of each nurse: one from the beginning of the work shift (between 7:30-8:30 a.m.) and one from the end of the work shift (between 1:00-2:00 p.m.).

Then the HRV data were analyzed using the Kubios HRV (version 2.0) software (http://kubios.uef.fi). Prior to computation of the HRV parameters, the very low frequency trend components (frequencies below $0.04 \mathrm{~Hz}$ ) were removed from the selected periods of HRV data by using a smoothness priors method ${ }^{27)}$. The time-domain parameters SDNN and RMSSD were computed from the detrended data, whereas meanRR was computed from the non-detrended data. Furthermore, prior to spectrum estimation, HRV data were interpolated ( $4 \mathrm{~Hz}$ cubic spline interpolation) in order to have equidistantly sampled data. The spectrum estimates were then computed by Welch's periodogram method (window width 256 seconds with $50 \%$ overlap).

\section{Questionnaires}

At baseline and follow-up, the subjects filled out a questionnaire with validated questions from the international Standard Shiftwork Index ${ }^{28}$, Work Ability Index (WAI) ${ }^{29,30)}$ and the Occupational Stress Questionnaire (OSQ) ${ }^{31)}$. The questionnaire included information about age, weight, height, health status (i.e., number of diseases diagnosed by a physician), work experience and shift-work experience. Body mass index (BMI, $\mathrm{kg} / \mathrm{m}^{2}$ ) was computed from self-reported weight and height. The perceived work ability was assessed using the total WAI score and the first item of the $\mathrm{WAI}^{29)}$. The range of the total WAI score is 7-49 points. Higher values indicate better perceived work ability. In the first item of the WAI, the current work ability was rated on a scale from 0 to 10 ranging from complete disability to work to work ability at its best during the occupational career.

Subjective work-related stress was assessed using one item of the Occupational Stress Questionnaire (OSQ): "Stress means the situation when a person feels tense, restless, nervous, or anxious, or is unable to sleep at night because his mind is troubled all the time. Do you feel that kind of stress these days?"31). The stress question assesses recent stress experience caused by work and other life situations. The item was rated on 5-point scale as follows: 1=very little, $2=$ fairly little, $3=$ somewhat, 4=fairly much and 5=very much. In the analyses, the two lowest values and two highest values were combined.

\section{Statistical analysis}

Statistical analyses were performed using SPSS (version 19.0, SPSS, Chicago IL, USA). Descriptive statistics (frequency, mean, standard deviation, ranges) were calculated. The linear mixed models were used to examine associations between HRV parameters and changes in shift work-schedules. Each HRV parameter (meanRR, SDNN, RMSSD, LF power, LF power in normalized units, HF power, $\mathrm{HF}$ in normalized units and $\mathrm{LF} / \mathrm{HF}$ ) was used as a dependent variable and analyzed separately. Age (in years) was used as a covariate in all analyses because it is known to have an influence ${ }^{32-34)}$. Time period (the beginning and the end of the work shift), shift schedule type (the conventional shift schedule at baseline or ergonomic shift schedules at follow-up) and the interaction between type of shift schedule and time period were used as factors in all analyses. The type of work shift (two-shift work or three-shift work) was used as a covariate in all analyses. Furthermore, the total WAI score and the value of the first item of the WAI (0-10) were covariates in separate analyses. The marital status and perceived work-related stress were included as separate factors in the analyses. Since there was no major influence of any of these factors (i.e., the total WAI score, the value of the first item of the WAI, marital status and perceived work related stress), they were excluded from the subsequent analyses. To account for the potential confounding effect of the previous work shift, the interaction between work shifts and type of work shift was tested by a linear mixed model. Furthermore, the possible age-related differences were analyzed in three age groups (20-39, 40-49 and 50-60 years) for interaction with the type of work shift. The distribution of residuals was verified in the analyses. $p$-values $<0.05$ were considered statistically significant.

\section{Results}

At the baseline, the mean age of the subjects was 45 years (SD 10, range 20-59), and their average employment duration in their current position was 22 years (Table 2). They had on average one physician-diagnosed disease (median 1, range 0-5).

Descriptive statistics of HRV parameters for the conventional (baseline) and ergonomic shift schedules (follow-up) are presented in Table 3. HRV parameters indicating parasympathetic activity (i.e., RMSSD and $\mathrm{HF}$ power in $\mathrm{ms}^{2}$ ) seemed to be somewhat higher at follow-up than at baseline, especially at the beginning of the shifts compared with at the end of the shifts.

In the comparison between the conventional and ergonomic shift schedules (Table 4), there were no significant differences in time-domain parameters at the beginning and end of the morning shift. Instead, 
Table 2. Means, standard deviations (SD) and ranges of the demographic, work ability and work experience characteristics at baseline $(n=48)$.

\begin{tabular}{lcc}
\hline Characteristic & Mean (SD, range) & $\mathrm{n}(\%)$ \\
\hline Age (years) & $45(10,20-59)$ & \\
Body Mass Index $\left(\mathrm{kg} / \mathrm{m}^{2}\right)$ & $25.5(4.2,17.9-35.6)$ & \\
Perceived work ability (WAI total score $\left.{ }^{2}\right)$ & $38.0(6.7,24.0-48.0)$ & \\
Perceived current work ability $(0-10)$ & $8(1,4-10)$ & \\
Work experience (years) & $22(10,1-36)$ & \\
Experience in shift work (years) & $17(9,1-34)$ & \\
Experience in current shift-work system (years) & $10(9,0-33)$ & $15(31)$ \\
Type of ward & & $33(69)$ \\
$\quad$ Acute & & $7(15)$ \\
Chronic & & $29(60)$ \\
Marital status & & $10(21)$ \\
Single & & $2(4)$ \\
Married & & \\
Married with children & & $21(44)$ \\
Single parent & & $16(33)$ \\
Work-related stress & & $11(23)$ \\
Very and fairly little & & \\
Somewhat & & \\
Fairly and very much & & \\
\hline
\end{tabular}

${ }^{a}$ WAI $=$ Work Ability Index.

Table 3. Mean values (SD and range) for the HRV parameters at the beginning and end of the morning shifts when the nurses were on a conventional shift-work schedule (baseline) and after implementation of the ergonomic shift schedules (follow-up, $\mathrm{n}=48$ ).

\begin{tabular}{|c|c|c|c|c|}
\hline \multirow[b]{3}{*}{ Parameter } & \multicolumn{2}{|c|}{ Baseline } & \multicolumn{2}{|c|}{ Follow-up } \\
\hline & Beginning of the shifts & End of the shifts & Beginning of the shifts & End of the shifts \\
\hline & Mean (SD, range) & Mean (SD, range) & Mean (SD, range) & Mean (SD, range) \\
\hline Mean RR (ms) & $687.9(126.7,476.7-1,176.8)$ & $730.7(118.3,545.1-1,044.7)$ & $715.2(126.1,509.9-1,049.4)$ & $745.7(113.5,509.9-1,006.4)$ \\
\hline SDNN (ms) & $24.4(7.7,7.8-40.2)$ & $26.7(7.5,7.7-38.9)$ & $24.5(8.7,6.6-47.5)$ & $26.6(8.8,9.2-48.3)$ \\
\hline RMSSD (ms) & $14.3(6.7,3.4-31.2)$ & $16.7(7.4,5.4-37.1)$ & $15.8(8.5,4.3-55.0)$ & $17.3(7.2,5.6-42.3)$ \\
\hline LF power $\left(\mathrm{ms}^{2}\right)$ & $387.1(230.2,23.8-1,090.1)$ & $428.2(258.9,31.8-1,050.5)$ & $363.3(237.3,20.8-990.3)$ & $459.0(337.2,15.8-1,491.2)$ \\
\hline LF power (n.u.) ${ }^{\mathrm{a}}$ & $83.6(9.6,46.4-97.3)$ & $79.1(14.6,19.0-94.8)$ & $78.2(14.0,26.4-93.5)$ & $78.5(13.0,44.1-95.6)$ \\
\hline HF power $\left(\mathrm{ms}^{2}\right)$ & $85.7(85.3,3.2-398.3)$ & $118.3(107.2,8.6-461.5)$ & $120.9(199.4,2.7-1,343.1)$ & $119.2(115.4,4.1-697.8)$ \\
\hline HF power (n.u.) $)^{b}$ & $16.4(9.6,2.7-53.6)$ & $20.9(14.6,5.2-81.0)$ & $21.8(14.0,6.5-73.6)$ & $21.5(13.0,4.4-55.9)$ \\
\hline $\mathrm{LF} / \mathrm{HF}$ & $7.5(6.3,0.9-36.2)$ & $6.0(4.3,0.2-18.2)$ & $5.5(3.7,0.4-14.4)$ & $5.6(4.1,0.8-21.7)$ \\
\hline
\end{tabular}

${ }^{a}$ n.u. $=$ normalized units LF / (total power - VLF). ${ }^{b}$ n.u.=normalized units HF / (total power - VLF). Mean RR=mean of beat-to-beat RR interval series. SDNN=standard deviation of all normal RR intervals. RMSSD=the root mean square of successive RR interval differences. $\mathrm{LF}=$ power of the low-frequency component (frequency range $0.04-0.15 \mathrm{~Hz}$ ). HF=power of the high-frequency component (frequency range $0.15-0.4 \mathrm{~Hz}$ ). $\mathrm{LF}=$ power of the very low frequency component (frequency range $0-0.04 \mathrm{~Hz}$ ).

the follow-up (i.e., ergonomic shift work schedule) values of frequency-domain parameters, including the normalized LF power and LF/HF ratio at the beginning of the shift, were reduced and the normalized HF power increased compared with the baseline (i.e., conventional shift schedule). These results point to a decline in sympathetic tone and increase in parasym- pathetic contribution to the heart rate, i.e., a decrease in the sympathovagal balance, for the ergonomic shift schedules (Table 4).

The analysis of interaction between shift schedule type (the conventional and ergonomic shift schedules) and the previous shift revealed a significant difference between the baseline and the follow-up when the 
Table 4. Age-adjusted differences in the HRV parameters between the conventional shift-work schedule (baseline) and ergonomic shift schedules (follow-up) at the beginning and end of the morning shifts.

\begin{tabular}{|c|c|c|c|c|}
\hline \multirow[b]{2}{*}{ Parameter } & \multicolumn{2}{|c|}{ Beginning of the shifts } & \multicolumn{2}{|l|}{ End of the shifts } \\
\hline & Mean difference $(95 \% \mathrm{CI})^{\mathrm{c}}$ & $p$-value ${ }^{\mathrm{d}}$ & Mean difference $(95 \% \mathrm{CI})^{\mathrm{c}}$ & $p$-value ${ }^{\mathrm{d}}$ \\
\hline Mean RR (ms) & $24.2(-13.8,62.3)$ & 0.210 & $15.5(-22.6,53.5)$ & 0.422 \\
\hline SDNN (ms) & $0.4(-2.1,2.8)$ & 0.761 & $0.4(-2.0,2.8)$ & 0.758 \\
\hline $\operatorname{RMSSD}(\mathrm{ms})$ & $1.3(-0.9,3.5)$ & 0.250 & $0.6(-1.6,2.8)$ & 0.596 \\
\hline LF power $\left(\mathrm{ms}^{2}\right)$ & $-11.4(-98.3,75.4)$ & 0.795 & $47.2(-39.6,134.1)$ & 0.284 \\
\hline LF power (n.u.) $)^{\mathrm{a}}$ & $-4.8(-9.2,-0.5)$ & 0.030 & $-0.2(-4.6,4.1)$ & 0.921 \\
\hline HF power $\left(\mathrm{ms}^{2}\right)$ & $32.6(-10.4,75.7)$ & 0.136 & $-0.1(-43.2,42.9)$ & 0.996 \\
\hline HF power (n.u. $)^{b}$ & $4.8(0.5,9.2)$ & 0.030 & $0.2(-4.1,4.6)$ & 0.921 \\
\hline $\mathrm{LF} / \mathrm{HF}$ & $-2.0(-3.8,-0.2)$ & 0.032 & $-0.4(-2.2,1.5)$ & 0.680 \\
\hline
\end{tabular}

${ }^{a}$ n.u.=normalized units, LF / (total power - VLF). ${ }^{b}$ n.u.=normalized units HF / (total power - VLF). ${ }^{c}$ Mean difference (95\% confidence Interval) between follow-up and baseline. ${ }^{\mathrm{d}}$ Linear mixed model with age, time period and type of work shift (two-shift work or three-shift work) as covariates. Mean RR=mean of beat-to-beat RR interval series. SDNN=standard deviation of all normal RR intervals. RMSSD=the root mean square of successive RR interval differences. $\mathrm{LF}=$ power of the low-frequency component (frequency range $0.04-0.15 \mathrm{~Hz}$ ). $\mathrm{HF}=$ power of the high-frequency component (frequency range $0.15-0.4 \mathrm{~Hz}$ ). LF=power of the very low frequency component (frequency range $0-0.04 \mathrm{~Hz}$ ).

morning shift was the previous shift. With the ergonomic shift schedules, when the previous shift was the morning shift, the RMSSD ( $p=0.001)$, HF power $(p=0.006)$ and normalized HF power $(p=0.006)$ were increased and the normalized LF power $(p=0.006)$ was decreased in comparison with the corresponding values with the conventional shift schedule.

The analysis of interaction between age and analyzed time periods (the beginning and the end of the work shift) revealed a significant interaction between age groups and meanRR. The changes in meanRR values during conventional and ergonomic shift schedules were higher in the two younger age groups (20-39 and 40-49 years) as compared with older nurses (age range 50-60), i.e., the meanRR values increased during shifts more among the two younger age groups than among the older nurses. There were no significant differences between age groups in the comparison between the shift schedules.

\section{Discussion}

This study of 48 nurses examined the psychophysiological strain related to conventional and ergonomic shift schedules using HRV analysis. Changes in HRV were investigated by analyzing time periods from the beginning and the end of the morning shift in conventional and ergonomic shift schedules. Through the Healthy Working Hours Research and Development Project, the main alteration occurred in the shifts from backward to forward rotation in order to increase recovery times by excluding the short free times in combinations of evening and morning shifts ${ }^{25}$. Our results indicated that a change from a conventional shift schedule to a more ergonomic version was associated with beneficial changes in HRV. The comparison between the beginning and the end of the shift with the nurses on the conventional shift schedule showed a higher meanRR and RMSSD at the end of the shift. In addition, normalized LF power was significantly lower and normalized HF power significantly higher at the end of shift. These results point to an increase in parasympathetic activation of the ANS occurring during the shift.

In the ergonomic shift schedules (follow-up), LF power was instead higher at the end of the shift indicating a possible increase in sympathetic activation of the ANS during the shift. This is not an unexpected result, since if as the subjects have the low values at the beginning of the work shift, they are likely to increase more as they start to work. On the other hand, the greater sympathetic activity may indicate more rush or work-related stressful events at the end of the shift. Hence this result may support the positive effects of the ergonomic schedule; even if the work shift was more stressful, the autonomic nervous system of the subjects was better able to recover before the next work shift.

The main aim of this study was to detect possible positive effects of the ergonomic shift arrangement on psychophysiological strain such as increased HRV or a decrease in the sympathovagal balance of the ANS. Prior studies have indicated that the implementation of ergonomic recommendations for shift work can have positive effects on sleep, alertness and overall subjective well-being 22,23). In the previous report of Hakola et al. $(2010)^{25)}$ with this same study group, more 
ergonomic working time arrangements (i.e., change from backward to forward rotation and increased recovery time between evening and morning shifts) had positive effects on the perceived physical, mental and social well-being of the nurses in all age groups. In the present prospective study, positive effects of ergonomic shift arrangements were detected on objective measurements of HRV. Our findings suggest that the beginning of the work shift was less stressful and the psychophysiological strain of nurses was lower or that the nurses had recovered better from the previous work shift when on the ergonomic shiftwork schedules when compared with the conventional one. The normalized LF power and LF/HF ratio both declined and normalized HF power increased when the nurses were working on the ergonomic shift schedules. Furthermore, the RMSSD and HF power values were increased with the ergonomic shift schedules compared with the conventional shift schedule, although the result was not statistically significant. These results point to a decrease in sympathetic tone and an increase in parasympathetic control of heart rate, i.e., a decrease in sympathovagal balance, with the ergonomic shift work schedule. The main alteration of the Healthy Working Hours Research and Development Project ${ }^{25)}$ was from backward to forward rotation even though the number of total working hours and the amounts of weekend, evening and night work remained the same. Thus, the nurses may have had a better opportunity to recover after the work shift, because of the increased time available for recovery between shifts. This may explain the positive effects on HRV, i.e., decrease in sympathovagal balance, acutely and in the long term with the ergonomic shift schedule.

Age affects $\mathrm{HRV}^{32-34)}$, hence all the analyses were controlled for age. However, our additional age-stratified analysis detected a trend for the older nurses (age group 50-60) to have lower changes in HRV values during work shifts than their younger colleagues (age groups 20-39 and 40-50 years), but the only significant difference between younger and older age groups was in meanRR values. Although these results merit caution due to the low number of subjects, lower HRV values may reflect increased sympathetic activation of the ANS, especially during work. One potential explanation may be that differences may be attributable to the adaptation of the nurses to shift work and by the healthy-worker effect ${ }^{35)}$. The older shift workers are likely to be a self-selected group, i.e., those who encounter few problems with working shifts tend to stay in shift work. Hence, this difference seems to be attributable to normal physiological changes (i.e., decreased maximal heart rate and stroke volume resulting in lowered cardiac volume) due to the ageing process ${ }^{36)}$.

One strength of the study was the prospective design. To the best of our knowledge, only a limited number of longitudinal studies of shift work have used HRV measures ${ }^{37)}$. Furthermore, we were able to measure HRV under actual working conditions, which has seldom been done in previous studies. Moreover, we were able to use objective psychophysiological measurements to study the effectiveness of shift schedule modifications. In earlier studies where HRV has been used at work, the focus has been on the comparison between shift work and daywork ${ }^{10,12-14,17,37)}$. The psychophysiological effects of changes to shift-work schedules have not been examined previously by using HRV recordings to the best of our knowledge.

HRV recordings from $\mathrm{HR}$ monitoring seem to be useful and provide an objective assessment of psychophysiological strain at work. However, HRV recordings need to be supplemented with subjective methods, as these can provide valuable information to help in the interpretation of the HRV results ${ }^{9,34,38)}$. In this regard, we controlled for the potential effect of perceived work-related stress or work ability on HRV parameters in our analyses, but there was no effect on psychophysiological strain in shift schedules. This may suggest that further studies would be warranted with more detailed subjective information or even larger sample sizes.

So far, no reference values for HRV parameters are available, although attempts to determine them have been made ${ }^{32-34)}$. Therefore, more longitudinal observations of the individual changes in HRV are essential to advance the reliability and predictive validity of the HRV method, for example, for application in occupational health care. However, by monitoring the HRV, the level of strain at work and recovery after work can be evaluated ${ }^{13,15)}$. One practical implication of HRV monitoring would be that the indicators of recovery from work would be useful for use in occupational health care in reducing psychophysiological strain from work and in early identification and prevention of stress. In particular, the information concerning work load and recovery obtained from HRV would help in planning work schedules and strategies for the assessment and promotion of health at work.

Examination of psychophysiological strain after work (i.e., during leisure time or sleep) based on the HRV would be essential in terms of recovery, because the negative effects of work-induced stress may be moderate after work. On the other hand, comparison between other time periods during leisure time or sleep would be influenced by various confounding effects, for example, smoking ${ }^{9)}$ or exercising ${ }^{9)}$. This would be very challenging to control in study settings, especially with a small sample size. In this study, 
the purpose was to use the most comparable time periods to examine the psychophysiological strain related to conventional and ergonomic shift schedules. The beginning of the morning shift was considered the most comparable time period, since the way the work time was organized allowed information about the previous work shift to be collected and hence controlled for in the analyses. Therefore, the beginning of the work shift would be the most suitable time period for analysis of the psychophysiological strain related to conventional and ergonomic shift schedules.

The increased time interval between shifts is important in terms of recovery from stress, but another interpretative factor would be how leisure time is spent. Psychological detachment from work (i.e., mentally switching off), relaxation-oriented strategies, mastery-oriented strategies and experience of control during leisure-time are known to affect the experience of recovery ${ }^{39)}$. Unfortunately, we were not able to take these factors into account in this study. On the other hand, the comparison between other time periods during leisure time or sleep would be influenced by the various confounding effects, for example, smoking or exercising. This would be very challenging to control in study settings, especially with small sample size. In this study, the purpose was to have the most comparable time periods to examine the psychophysiological strain related to conventional and ergonomic shift schedule. We assumed that the beginning of the work shift is a comparable and somewhat controlled (i.e., every subject in the same kind of situation) time period and therefore suitable for analysis of the psychophysiological strain related to conventional and ergonomic shift schedules.

One limitation of the study is that there may have been additional confounding due to factors such as timing of the previous work shift, quality of sleep during the night before the measurements, and stressful situations in life. Another limitation of the study was the lack of information concerning caffeine intake or smoking during the analyzed work shifts. However, throughout the 24-hour recording periods, the subjects were instructed to work in a habitual manner and to maintain their normal life style, including possible smoking and caffeine intake, which might affect HRV. However, the prospective study setting in which each subject served as her own control is likely to have minimized effects of these differences. Furthermore, we included perceived occupational stress in the models to control for the putative effect of stress on $\mathrm{HRV}^{40)}$. However, the effect of occupational stress was very minor, which means that the changes in HRV must be attributable to factors other than stress at work. In addition, the sample size of the study was relatively small. However, a comparison with other HRV studies of shift work $^{10,12,14,37)}$ indicates that the sample size of this study is fully comparable with the other studies and hence can be considered adequate.

In conclusion, it seems that the psychophysiological strain based on the analysis of HRV was lower at the beginning of the work shift with the ergonomic shiftwork schedules compared with the conventional shift schedule. This indicates that the ergonomic shift-work schedule has a positive effect on the ANS recovery that should take place between successive work shifts.

Acknowledgments: The Healthy Working Hours Research and Development Project was funded by the Finnish Work Environment Fund [project number 105273] and by the city of Helsinki (Health Centre and Human Resources Centre). This work was supported by the Finnish Work Environment Fund [project number 110005]. M.P.T. and P.A.K. were supported by Academy of Finland grants [project numbers 126873, 123579].

\section{References}

1) Härmä M. Workhours in relation to work stress, recovery and health. Scan J Work Environ Health 2006; 32: 502-14.

2) Åkerstedt T, Wright Jr KP. Sleep loss and fatigue in shift work and shift work disorder. Sleep Med Clin 2009; 4: 257-71.

3) Puttonen S, Härmä M, Hublin C. Shift work and cardiovascular disease-pathways from circadian stress to morbidity. Scand J Work Environ Health 2010; 36: 96-108.

4) Vogel M, Braungardt T, Meyer W, Schneider W. The effects of shift work on physical and mental health. J Neural Transm 2012; 119: 1121-32.

5) Vyas M, Garg A, Iansavichus A, et al. Shift work and vascular events: systematic review and metaanalysis. BMJ 2012; 26; 345: e4800.

6) Puttonen S, Viitasalo K, Härmä M. The relationship between current and former shift work and the metabolic syndrome. Scand J Work Environ Health 2012; 38: 343-8.

7) de Castro AB, Fujishiro K, Rue T, Tagalog EA, Samaco-Paquiz LP, Gee GC. Associations between work schedule characteristics and occupational injury and illness. International Nursing Review 2010; 57: 188-94.

8) Task Force of the European Society of Cardiology the North American Society of Pacing and Electrophysiology. Heart rate variability. Standards of measurement, physiological interpretation, and clinical use. Circulation 1996; 93: 1043-65.

9) Acharya UR, Joseph KP, Kannathal N, Lim CM, Suri JS. Heart rate variability: a review. Med Bio Eng Comput 2006; 44: 1031-51.

10) Ito H, Nozaki M, Maruyama T, Kaji Y, Tsuda Y. 
Shift work modifies the circadian patterns of heart rate variability in nurses. Int $\mathrm{J}$ Cardiol 2001; 79; 231-6.

11) Rajaratnam $S$, Arendt J. Health in a 24-h society. Lancet 2001; 358: 999-1005.

12) Ishii N, Dakeishi M, Sasaki M, Iwata T, Murata K. Cardiac autonomic imbalance in female nurses with shift work. Auton Neurosci 2005; 122: 94-9.

13) Van Amelsvoort LG, Schouten EG, Maan AC, Swenne CA, Kok FJ. Occupational determinants of heart rate variability. Int Arch Occup Environ Health 2000; 73: 255-62.

14) Chung MH, Kuo TB, Hsu N, Chu H, Chou KR, Yang CC. Sleep and autonomic nervous system changes - enhanced cardiac sympathetic modulations during sleep in permanent night shift nurses. Scan J Work Environ Health 2009; 35: 180-7.

15) Wehrens $S$, Hampton $S$, Skene D. Heart rate variability and endothelial function after sleep deprivation and recovery sleep among male shift and non-shift workers. Scand J Work Environ Health 2012; 38: 171-81.

16) Thayer JF, Yamamoto SS, Brosschot. The relationship of autonomic imbalance, heart rate variability and cardiovascular disease risk factors. Int $\mathrm{J}$ Cardiol 2010; 141: 122-31.

17) Collins SM, Karasek RA, Costas K. Job strain and autonomic indices of cardiovascular disease risk. Am J Ind Med 2005; 48: 182-93.

18) Su TC, Lin LY, Baker D, et al. Elevated blood pressure, decreased heart rate variability and incomplete blood pressure recovery after a 12-hour night shift work. J Occup Health 2008; 50: 380-6.

19) Lo SH, Lin LY, Hwang JS, Chang YY, Liau CS, Wang JD. Working the night shift causes increased vascular stress and delayed recovery in young women. Chronobiol Int 2010; 27: 1454-68.

20) Knauth P. Changing schedules: shiftwork. Chronobiol Int 1997; 14: 159-71.

21) Knauth P, Hornberger S. Preventive and compensatory measures for shift work. Occup Med 2003; 53: 109-16.

22) Härmä M, Hakola T, Kandolin I, et al. A controlled intervention study on the effects of a very rapidly forward rotating shift system on sleep-wakefulness and well-being among young and elderly shift workers. Int J Psychophysiol 2006; 59: 70-9.

23) Hakola T, Härmä M. Evaluation of a fast forward rotating shift schedule in the steel industry with a special focus on ageing and sleep. J Hum Ergol 2001; 30: 315-9.

24) Joyce K, Pabayo R, Critchley JA, Bambra C. Flexible working conditions and their effects on employee health and wellbeing (Review). The Cochrane Library. Cochrane Database Syst Rev 2010; 2: CD008009

25) Hakola $T$, Paukkonen $M$, Pohjonen T. Less quick returns-Greater well-being. Ind Health 2010; 48: 390-4.
26) Achten J, Jeukendrup AE. Heart rate monitoring. applications and limitations. Sports Med 2003; 33: 517-38.

27) Tarvainen MP, Ranta-aho PO, Karjalainen PA. An advanced detrending method with application to HRV analysis. IEEE Trans Biomed Eng 2002; 49: $172-5$

28) Tucker P, Knowles SR. Review of studies that have used the Standard Shiftwork Index: evidence for the underlying model of shiftwork and health. Applied Ergonomics 2008; 39: 550-64.

29) Tuomi K, Ilmarinen J, Eskelinen L, Järvinen E, Toikkanen J, Klockars M. Prevalence and incidence rates of diseases and work ability in different work categories of municipal occupations. Scan J Work Environ Health 1991; 17: 67-74

30) De Zwart BC, Frings-Dresen MH, van Duivenbooden JC. Test-retest reliability of the Work Ability Index questionnaire. Occup Med 2002; 52: 177-81.

31) Elo AL, Leppänen A, Jahkola A. Validity of a single-item measure of stress symptoms. Scan J Work Environ Health 2003; 29: 444-51.

32) Umetani K, Singer DH, McCraty R, Atkinson M. Twenty-four hour time domain heart rate variability and heart rate: relations to age and gender over nine decades. J Am Coll Cardiol 1998; 31: 593-601.

33) Bonnemeier H, Richardt G, Potratz J, et al. Circadian profile of cardiac autonomic nervous modulation in healthy subjects: differing effects of aging and gender on heart rate variability. J Cardiovasc Electrophysiol 2003; 14: 791-9.

34) Koskinen T, Kähönen M, Jula A, et al. Short-term heart rate variability in healthy young adults: the cardiovascular risk in young Finns study. Auton Neurosci 2009; 145: 81-8.

35) Nagaya $T$, Yoshida H, Takahashi H, Kawai $M$. Markers of insulin resistance in day and shift workers aged 30-59 years. Int Arch Occup Environ Health 2002; 75: 562-8.

36) Kenny GP, Yardley JE, Martineau L, Jay O. Physical work capacity in older adults: implications for the aging worker. Am J Ind Med 2008; 51: 610-25.

37) Togo F, Takahashi M. Heart rate variability in occupational health-A systematic review. Ind Health 2009; 47: 589-602.

38) Buchheit M, Gindre C. Cardiac parasympathetic regulation: respective associations with cardiorespiratory fitness and training load. Am J Physiol Heart Circ Physiol 2006; 291: 451-8.

39) Sonnentag S, Fritz C. The recovery experience questionnaire: development and validation of a measure for assessing recuperation and unwinding from work. J Occup Health Psychol 2007; 3: 204-21.

40) Thayer JF, Åhs F, Fredrikson M, Sollers JJ, Wager TD. A meta-analysis of heart rate variability and neuroimaging studies: implications for heart rate variability as a marker of stress and health. Neurosci Biobehav Rev 2012; 36: 747-56. 